\title{
Factores que pueden influir en el rendimiento académico de estudiantes de Bioquímica que ingresan en el programa de Medicina de la Universidad del Rosario-Colombia
}

\author{
R. Garzón, M.O. Rojas, L. del Riesgo, M. Pinzón, A.L. Salamanca
}

Introducción. El análisis del rendimiento académico de los estudiantes que ingresan a una institución de educación superior, así como los factores que pueden estar influyendo en él, genera respuestas a los interrogantes que corrientemente se hacen las personas involucradas en los procesos educativos y permite proponer soluciones para mejorar el desempeño de los estudiantes universitarios. Sujetos y métodos. Se determinó la asociación entre el rendimiento académico de los estudiantes en el primer nivel de Bioquímica, con los factores de tipo académico y demográfico; se utilizaron dos métodos de análisis: el primero, numérico, de acuerdo con los promedios de las notas parciales y finales; el segundo, categorizado como éxito $(\geq 3,0)$ o fracaso $(<3,0)$, de acuerdo con el promedio de la nota definitiva de la asignatura. Resultados. Se encontró una asociación positiva y estadísticamente significativa entre los resultados de las pruebas de estado generales y específicas (ciencias naturales y matemáticas), el estatus de becario institucional y el ingreso directo a la carrera sin realizar cursos preuniversitarios, con el rendimiento académico en Bioquímica. Factores como el colegio en el cual realizaron la educación secundaria y la ciudad de procedencia no afectaron significativamente el rendimiento en ninguno de los análisis aplicados. Conclusiones. El rendimiento académico en Bioquímica se ve afectado directamente por factores que involucran las competencias individuales de los estudiantes, pero no por factores demográficos

Palabras clave. Aprendizaje activo. Competencias en educación media. Factores de ingreso en la universidad. Rendimiento académico.

Factors that influence the academic performance in Biochemistry students who have started the Medicine program of Rosario University-Colombia

Introduction. The analysis of the students' academic achievement in a higher education institution together with the dif- ferent factors that affect the student's performance generates answers to the questions that the people involved in the teaching process commonly ask themselves. This analysis is therefore, an excellent way to find and propose solutions in order to improve the student's performance. Subjects and methods. The association between the students' academic performance and the academic and demographic factors was determined in the first level of Biochemistry. Two methodologies were used for this purpose. The first one, a numerical analysis based on the partial and final grades, and a second categorical method based on the final success ( $\geq 3.0$ ) or failure $(<3.0)$ on the subject. Results. A positive and statistically significant correlation was found when the academic performance in Biochemistry, the general and specific results of the state test (natural sciences and mathematics), the institutional scholarship status and entering the Medicine program without pre-university courses was analyzed. Factors, such as coming from different schools and the city of origin, do not affect significantly the performance in any of the analyses. Conclusions. Academic performance in Biochemistry is directly affected by factors involving the individual student's skills while the demographical factors are not involved.

Key words. Academic performance. Active learning, Intellectual competence. Learning influential factors. Teaching process.

\section{Introducción}

'Una de las dimensiones más importantes en el proceso de enseñanza y aprendizaje lo constituye el rendimiento académico del estudiante' [1]; este rendimiento, desempeño o aptitud escolar es, como postula Jiménez [2], un nivel de conocimientos demostrado en una área o materia comparado con la norma de edad y nivel académico, o según Spinola [3], 'el cumplimiento de las metas,
Facultad de Medicina y Facultad de Ciencias Naturales y Matemáticas. Unidad de Bioquímica. Universidad del Rosario. Bogotá, Colombia.

Correspondencia Dra. María Orfa Rojas. Universidad del Rosario Facultad de Ciencias Naturales y Matemáticas. Unidad de Bioquímica. Ctra. 24, n. . 63, C-69 Bogotá, Colombia.

E-mail mariaorfarojas@yahoo.com

Agradecimientos Al Sr. Milciades Ibáñez, por su asesoría estadística. 
logros u objetivos establecidos en el programa o asignatura que está cursando el estudiante'.

Al evaluar el rendimiento académico y los procedimientos para mejorarlo, se analizan en mayor o menor grado los factores que pueden influir en él, considerando generalmente los personales (motivaciones, capacidades cognitivas, hábitos de estudio, conocimientos previos, nivel de pensamiento formal, personalidad, autoestima, desarraigo, habilidad social, inteligencia emocional), los relacionados con el entorno familiar y social en el que se desenvuelve el estudiante (violencia, adicciones, desempleo, inseguridad), los dependientes de la institución (material didáctico, equipamiento, relación docentes/estudiantes, políticas de ingreso, planes de estudios actualizados) y los que dependen de los docentes (metodología de enseñanza, motivaciones para enseñar, capacitación, uso de nuevas tecnologías) [4].

De acuerdo con esto, existen diversas investigaciones que se dirigen a encontrar explicaciones al bajo rendimiento académico, las cuales van desde estudios exploratorios, descriptivos y correlacionales hasta estudios explicativos [5].

Algunos autores coinciden en afirmar que la inteligencia es uno de los factores que influyen con más peso en la predicción del rendimiento y que el uso de instrumentos estandarizados (tests) ayudaría a detectar grupos con riesgo de fracaso escolar [6], mientras que otros afirman que, aunque es muy complejo ponderar la influencia específica de cada uno de los posibles factores involucrados, son las actitudes que mantienen los estudiantes hacia el aprendizaje una de las variables fundamentales que influyen en los resultados escolares. A este respecto, los autores llaman la atención acerca del supuesto de que, pese a que todos los estudiantes que ingresan en la universidad poseen actitudes muy favorables hacia el estudio, muchos de ellos obtienen resultados deficientes [7].

Por otro lado, otros estudios plantean que los exámenes de ingreso a la educación superior en los cuales se analizan habilidades que implican razonamiento analítico, verbal y numérico, muestran un alto grado de validez predictiva para el buen desempeño de los estudiantes [8,9]. En Colombia, los exámenes de estado han sido una herramienta para que los estudiantes procedentes de la educación media estén informados de sus competencias en las áreas evaluadas y tengan elementos para orientar sus opciones profesionales, y por otra parte, para que las universidades tengan criterios de selección de los aspirantes a los diferentes programas académicos que ellas imparten (www.icfes.gov.co).

Lo anterior muestra que el rendimiento de los estudiantes se ha venido analizando desde la escuela hasta la universidad, y que en ésta última es una medida de calidad para su permanencia.

El rendimiento académico como fenómeno multifactorial evidencia que se pueden tener adecuadas capacidades intelectuales y aptitudes, pero esto no necesariamente conlleva un rendimiento académico adecuado [2], con lo cual se puede concluir que 'el rendimiento escolar del estudiante depende del dominio del principal de los conocimientos: aprender a aprender' [10].

Una primera aproximación a la valoración del rendimiento académico en la Universidad del Rosario forma parte de un estudio realizado por Vélez y Roa [11], en el cual se demostró la asociación del fracaso académico o pérdida de cupo con algunos factores como: la falta de lectura como pasatiempo, la vivencia de violencia intrafamiliar, el consumo de sustancias psicoactivas y alcohol, el tipo de colegio en el que se cursó la educación media, la omisión de estudios preparatorios para el ingreso en la universidad, el tipo de dependencia económica y las notas de las asignaturas fundamentales del primer período de la carrera de Medicina (biología y bioquímica), así como de los promedios trimestrales en el mismo período.

Posteriormente, el grupo de profesores de Bioquímica de la Facultad de Medicina de la misma universidad consideró, al igual que muchos investigadores en educación, que las estrategias pedagógicas tienen una influencia definitiva en la apropiación del conocimiento, pero que además pueden utilizarse como herramientas de formación y que la apropiación de conocimientos debe medirse principalmente a través de la capacidad de análisis desarrollada por el estudiante y de su destreza para lograr aplicar sus conocimientos a la solución de problemas cotidianos [12,13]. Por ello, a partir del segundo semestre de 2005, este grupo dio inicio a una nueva metodología para el aprendizaje de la Bioquímica, abriendo paso a la utilización de estrategias pedagógicas de aprendizaje activo que incluyeron la participación tanto individual como en grupo del estudiante en su proceso de aprendizaje [12-14]; para ello, se propició el análisis y apropiación de conceptos a tra- 
vés de su comprensión previa mediante lecturas individuales, seguida de la discusión en grupo, la experimentación como herramienta para afianzar los conceptos en estudio, la lectura y análisis de la literatura científica para la comprensión de situaciones de salud y enfermedad, y el análisis de situaciones problemáticas y la aplicación de conceptos a la solución de las mismas como sistema individual y grupal de evaluación académica $[12,13]$. En este trabajo se analizaron otros factores que, a juicio del grupo de profesores, afectan el rendimiento académico de los estudiantes, como el análisis de las competencias individuales de ingreso a la universidad, la presión de ser beneficiarios de becas que implican mantener promedios semestrales por encima de 4,0, o el cambio en su entorno familiar al tener que realizar sus estudios en una ciudad diferente a la que habitaron antes de ingresar a la universidad.

\section{Sujetos y métodos}

\section{Muestra}

Se compone de 630 estudiantes que ingresaron al programa de Medicina en la Universidad del Rosario a partir del primer semestre del 2005 (cohorte I), hasta el primer semestre del 2008 (cohorte VI) y que cursaron el primer nivel de Bioquímica.

El programa de medicina definido por la institución y aprobado por el Ministerio de Educación Nacional se ofrece semestralmente y, dentro de él, la Bioquímica es uno de los cursos que deben tomarse al iniciar el mismo, sin que existan prerrequisitos diferentes a los establecidos en los parámetros de ingreso a la universidad; la puntuación de los exámenes de Estado y la cualificación del estudiante en una entrevista grupal constituyen dichos parámetros. Quienes no logran superarlos, tienen la opción de hacerlo a través de los cursos preuniversitarios, dentro de los cuales está definido el nivel de desempeño que deben alcanzar para lograr la admisión al programa.

\section{Metodología}

Se analizó el rendimiento académico de los estudiantes de Bioquímica de primer semestre de cada una de las seis cohortes escogidas de acuerdo con el promedio de las calificaciones de sus tres notas parciales y de las notas definitivas resultantes de promediar las anteriores; cada una de las notas parciales comprendió dos evaluaciones, una individual y otra grupal. Ambos tipos de evaluaciones se centraron en el análisis y la aplicación de conceptos a la solución de problemas [13]. En el trabajo de grupo se enfatizó la comprensión de la literatura científica relativa al tema en estudio mediante la resolución cooperativa de problemas que exigían utilizar el conocimiento encontrado en el artículo científico seleccionado por los profesores y leído previamente por cada estudiante.

Se escogieron, como factores que pudieran estar influyendo en el rendimiento académico de los estudiantes (variables independientes), los resultados de los exámenes de Estado (Instituto Colombiano para el Fomento de la Educación Superior, ICFES), la categoría del colegio según el ICFES, la categoría de ingreso a la universidad -directa (resultados del ICFES $\geq 55$ puntos, y entrevista) o a través de un curso de preparación preuniversitaria (aquellos que no alcanzaron la puntuación requerida en el examen del ICFES, pero que superaron la entrevista)-, el ser beneficiario o no de beca por la universidad (el beneficio oscila entre el $10 \mathrm{y}$ el $100 \%$ del costo de la matrícula de acuerdo con las diferentes ofertas de la universidad) y el lugar de procedencia de los estudiantes (la misma ciudad sede de la universidad, u otras ciudades o municipios del país).

Teniendo en cuenta los factores seleccionados, se analizaron sus posibles efectos sobre el rendimiento académico en Bioquímica para todas las cohortes; en primer lugar se hizo un análisis numérico del rendimiento académico de acuerdo con los promedios de las calificaciones parciales y definitivas y en segundo, un análisis categorizado de acuerdo con la posibilidad de éxito $(\geq 3,0)$ o fracaso $(<3,0)$ en el promedio de la calificación definitiva de la asignatura.

\section{Análisis estadístico}

Para realizar las comparaciones entre las variables analizadas se utilizó el programa SPSS v. 16. De acuerdo con la distribución de la muestra, se analizaron los coeficientes de correlación de Sperman y las pruebas de Kruskal-Wallis y 


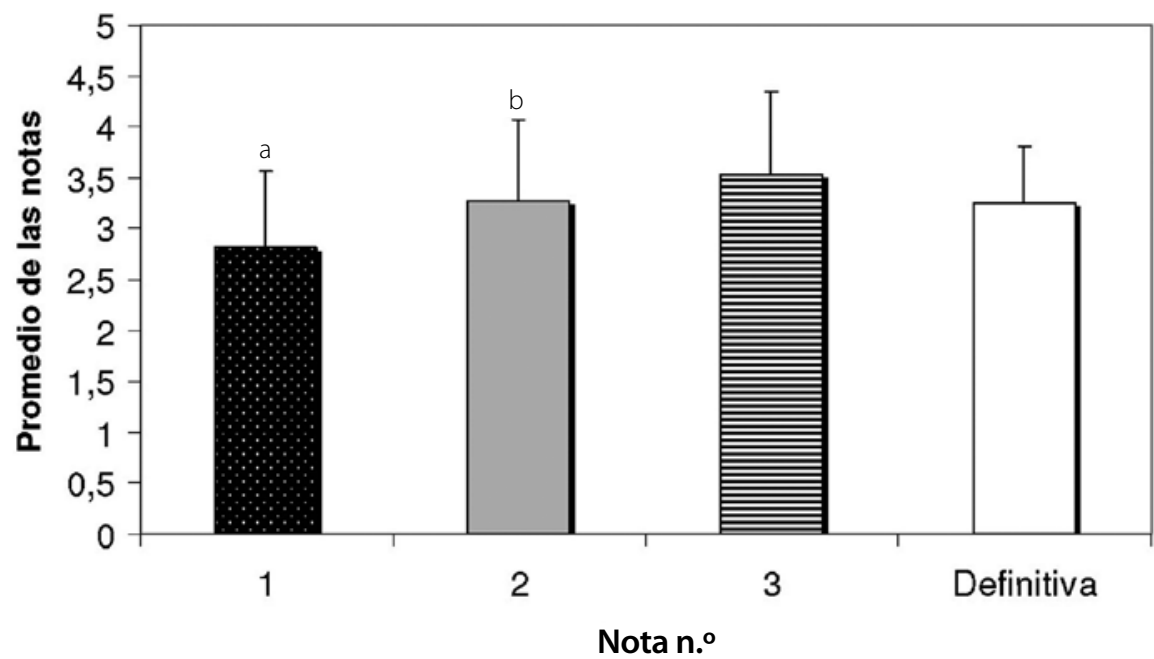

Figura 1. Rendimiento promedio en las notas de bioquímica para todas las cohortes. ${ }^{a}$ Significativamente diferente de las otras notas; ${ }^{b}$ Significativamente diferente comparándola con la tercera nota $(p<0,05)$.

Mann-Whitney para definir rangos promedio, fijando un intervalo de confianza del 95\%.

\section{Resultados}

\section{Análisis numérico del rendimiento académico}

El rendimiento académico de los estudiantes de Bioquímica en todas las cohortes estudiadas, visto como los promedios de las calificaciones en cada una de los tres períodos evaluativos y su definitiva $(2,8 \pm 0,7,3,3 \pm 0,8,3,5 \pm 0,8$ y $3,2 \pm 0,6$, respectivamente), muestra una tendencia a mejorar de acuerdo con el avance del semestre, observando diferencias significativas entre la primera nota y las demás, y entre la segunda y la tercera (Fig. 1).

Al comparar los resultados obtenidos entre las cohortes se encontró que hay diferencias significativas $(p<0.05)$ entre las cohortes IV y V (2007-I y 2007-II) y entre la última cohorte (2008-I) y las cohortes III y V $(p<0,05)$, cuyo ingreso se dio en los segundos semestres del año (2006-II y 2007-II) (Fig. 2). Las diferencias estadísticas demuestran la tendencia a obtener mejores resultados en aquellos grupos de estudiantes que ingresan en los segundos semestres del año, siendo más notoria en el último semestre anali- zado; es importante precisar que, para esta cohorte, adicionalmente se introdujo una estrategia de apoyo extraclase enfocada al afianzamiento de conceptos y a su aplicación a la solución de problemas, con la cual se había demostrado un efecto positivo sobre la mejora del rendimiento académico y el desarrollo de valores tales como la responsabilidad y la autonomía [13].

El análisis de las variables, teniendo en cuenta las diferentes categorías establecidas, mostró que el más alto porcentaje de estudiantes admitidos $(99,4 \%)$ se ubica en el rango medio de los resultados del examen de Estado (31-70 puntos sobre 100), que la mayoría proviene de colegios categorizados por el ICFES como muy superior (74,6\%), que el mayor porcentaje ingresó de forma directa a la universidad $(86,3 \%)$, que aproximadamente la mitad de quienes han ingresado han cubierto sus gastos de matrícula mediante becas parciales, y por último, que el mayor porcentaje vive en la ciudad donde se encuentra ubicada la universidad o muy cerca de ella $(73,7 \%)$, como se muestra en la tabla I.

Los resultados de los exámenes de Estado para todas las cohortes mostraron un promedio de 57,3 \pm 4,6 en el promedio general. En el caso de los resultados para biología, química, física y matemáticas, esos valores correspondieron a $60,2 \pm 6,1,55,7 \pm 5,8,55,7 \pm 7,4$ y $57,1 \pm 10,2$, 
Tabla I. Distribución de estudiantes de acuerdo con las variables analizadas.

\begin{tabular}{|c|c|c|c|}
\hline & & N. ${ }^{\circ}$ de estudiantes & Estudiantes dentro de la categoría (\%) \\
\hline \multirow{3}{*}{ Resultados del ICFES } & Bajo & 0 & 0,0 \\
\hline & Medio & 626 & 99,4 \\
\hline & Alto & 4 & 0,6 \\
\hline \multirow{5}{*}{$\begin{array}{l}\text { Categoría del colegio } \\
\text { (ICFES) }\end{array}$} & Bajo & 2 & 0,3 \\
\hline & Medio & 10 & 1,6 \\
\hline & Alto & 32 & 5,1 \\
\hline & Superior & 115 & 18,3 \\
\hline & Muy superior & 470 & 74,6 \\
\hline \multirow{2}{*}{ Categoría de ingreso } & Directa & 544 & 86,3 \\
\hline & Preuniversitario & 85 & 13,5 \\
\hline \multirow{2}{*}{ Beca } & Sí & 318 & 50,5 \\
\hline & No & 312 & 49,5 \\
\hline \multirow{2}{*}{ Ciudad de procedencia } & Capital & 464 & 73,7 \\
\hline & Otros municipios & 166 & 26,3 \\
\hline
\end{tabular}

respectivamente. Lo anterior mostró que los estudiantes que ingresan en la Universidad del Rosario están en el rango medio-alto de la población estudiantil colombiana, de acuerdo con el promedio de las puntuaciones del ICFES tanto generales como específicas.

Los resultados de los coeficientes de correlación de Sperman al cruzar los resultados del ICFES para cada una de las áreas, con respecto a las calificaciones parciales y las definitivas en Bioquímica, mostraron una asociación positiva $(p>0)$, aunque moderada, con valores entre 0,105 y 0,422 , en los cuales se evidenciaron diferencias estadísticamente significativas $(\mathrm{p}<0,01)$, como se observa en la tabla II.

La categoría de ingreso y de becario mostró diferencias significativas; la categorización del colegio según el ICFES mostró una tendencia positiva de los estudiantes cuyos colegios estuvieron en categoría superior y muy superior, pero sin ser significativamente diferente, mientras que la ciudad de origen de los estudiantes no mostró diferencia estadística al compararse con sus rendimientos académicos (Tabla III).

\section{Análisis categorizado del rendimiento académico}

Durante el período analizado, la categoría denominada 'éxito' mostró que el $76 \%$ de los estudiantes en promedio aprobaron la materia, mientras que el $24 \%$ de los estudiantes en promedio se clasificó en la categoría denominada 'fracaso'.

Los resultados del ICFES mostraron una ma- 
Tabla II. Correlación entre las notas promedio para las diferentes disciplinas de las ciencias naturales y los resultados del ICFES.

\begin{tabular}{cccccc}
\hline Notas & Promedio general & Biología & Química & Física & Matemáticas \\
\hline $1 .^{\mathrm{a}}$ & $0,219^{\mathrm{a}}$ & $0,202^{\mathrm{a}}$ & $0,219^{\mathrm{a}}$ & $0,144^{\mathrm{a}}$ & $0,156^{\mathrm{a}}$ \\
\hline $2 .^{\mathrm{a}}$ & 0,064 & 0,068 & $0,105^{\mathrm{a}}$ & $0,120^{\mathrm{a}}$ & $0,118^{\mathrm{a}}$ \\
\hline $3 .^{\mathrm{a}}$ & $0,422^{\mathrm{a}}$ & $0,198^{\mathrm{a}}$ & $0,263^{\mathrm{a}}$ & $0,171^{\mathrm{a}}$ & $0,272^{\mathrm{a}}$ \\
\hline Definitiva & $0,333^{\mathrm{a}}$ & $0,208^{\mathrm{a}}$ & $0,262^{\mathrm{a}}$ & $0,200^{\mathrm{a}}$ & $0,246^{\mathrm{a}}$ \\
\hline
\end{tabular}

a La correlación es significativa al nivel 0,01 (bilateral).

Tabla III. Rangos promedio y significación estadística de las notas definitivas para las variables analizadas.

\begin{tabular}{|c|c|c|c|c|c|}
\hline & & $n$ & $\begin{array}{l}\text { Rango } \\
\text { promedio }\end{array}$ & $\begin{array}{l}\text { Sig. asintót. } \\
\text { (bilateral) }\end{array}$ & $\begin{array}{l}\text { Prueba } \\
\text { estadística }\end{array}$ \\
\hline \multirow{3}{*}{$\begin{array}{l}\text { Categoría del colegio } \\
\text { (ICFES) }\end{array}$} & Bajo, medio, alto & 44 & 289,11 & \multirow{3}{*}{0,531} & \multirow{3}{*}{ Light } \\
\hline & Superior & 115 & 325,23 & & \\
\hline & Muy superior & 469 & 314,25 & & \\
\hline \multirow{2}{*}{ Categoría de ingreso } & Directa & 544 & 384,62 & \multirow{2}{*}{0,000} & \multirow{2}{*}{ Mann-Whitney } \\
\hline & Preuniversitario & 85 & 226,71 & & \\
\hline \multirow{2}{*}{ Beca } & $\mathrm{Si}$ & 318 & 375,48 & \multirow{2}{*}{0,000} & \multirow{2}{*}{ Mann-Whitney } \\
\hline & No & 311 & 253,16 & & \\
\hline \multirow{2}{*}{ Ciudad de procedencia } & Capital & 463 & 312,09 & \multirow{2}{*}{0,502} & \multirow{2}{*}{ Mann-Whitney } \\
\hline & Otros municipios & 166 & 323,12 & & \\
\hline
\end{tabular}

yor diferencia entre las medias en el promedio general así como en las cuatro áreas de interés, favorable para los que aprobaron (éxito) a diferencia de los que suspendieron (fracaso) la asignatura (Tabla IV), demostrando que unos resultados cuantitativos más favorables con respecto a la apropiación del conocimiento en las áreas disciplinares básicas, como la biología, la quími- ca, la física y las matemáticas, conduce necesariamente a los mejores resultados encontrados y, por consiguiente, al mejor aprovechamiento de la bioquímica.

El ser beneficiario o no de una beca institucional mostró diferencias significativas en los resultados de los estudiantes que aprobaron con respecto a los que suspendieron $(p<0,000)$; 


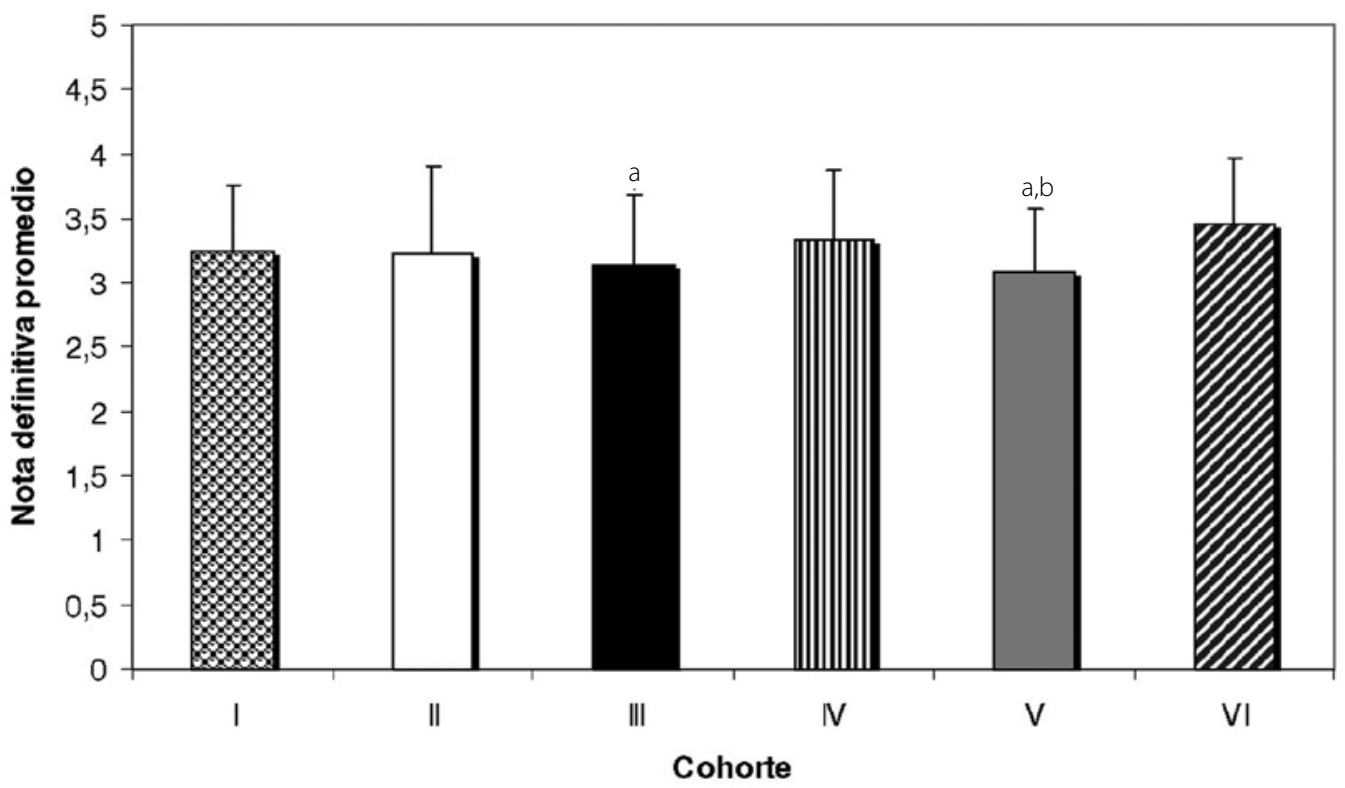

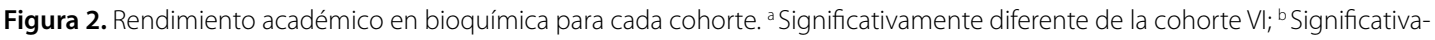
mente diferente de la cohorte IV $(p<0,05)$.

igual comportamiento se observó en los estudiantes que aprobaron la asignatura y que entraron de forma directa con respecto a los que realizaron estudios preuniversitarios $(p<0,004)$ (Fig. 3). Por otra parte, la categoría del colegio dada por el ICFES muestra una tendencia a favorecer los resultados del éxito de los estudiantes (59\%: bajo, medio, alto; $68 \%$ : superior; $73 \%$ : muy superior), pero sin arrojar diferencias significativas entre ellos. La ciudad de procedencia de los estudiantes no influyó significativamente en los resultados.

\section{Discusión}

Una de las variables más empleadas o consideradas por el sistema educativo para aproximarse al análisis del rendimiento académico de los estudiantes son las calificaciones escolares; se constituyen, por una parte, en un indicador predictivo del dominio de habilidades y conocimientos en las evaluaciones o exámenes en las distintas áreas, que el sistema considera necesarias y suficientes para su desarrollo como miembro activo de la sociedad [6].
$\mathrm{Al}$ analizar los resultados con base en las categorías definidas como éxito y fracaso, se observó que el promedio de las calificaciones definitivas, en los tres años del estudio, refleja una 'mortalidad académica' en la asignatura del $24 \%$, lo cual representa una disminución considerable si se tienen en cuenta los valores comunicados en años anteriores, en los cuales se alcanzó hasta un $34 \%$ de fracaso; hemos demostrado mediante el análisis de por lo menos cinco cohortes con las cuales se han manejado metodologías de aprendizaje pasivo y otras cinco que utilizaron las metodologías de aprendizaje activo implementadas para la apropiación de conocimientos en Bioquímica a partir del 2005, que estos últimos desarrollan competencias de trabajo individual y en grupo, de análisis y de crítica, entre otros, que les permiten una mayor posibilidad de éxito en la resolución de problemas cotidianos, lo cual se ha reflejado en mejores rendimientos académicos [resultados en prensa].

En todas las cohortes analizadas numéricamente, los resultados de los promedios de las calificaciones en los diferentes períodos evaluativos mostraron una mejoría estadísticamente significativa a medida que transcurría el semestre (Fig. 1). Sin embargo, la calificación definiti- 


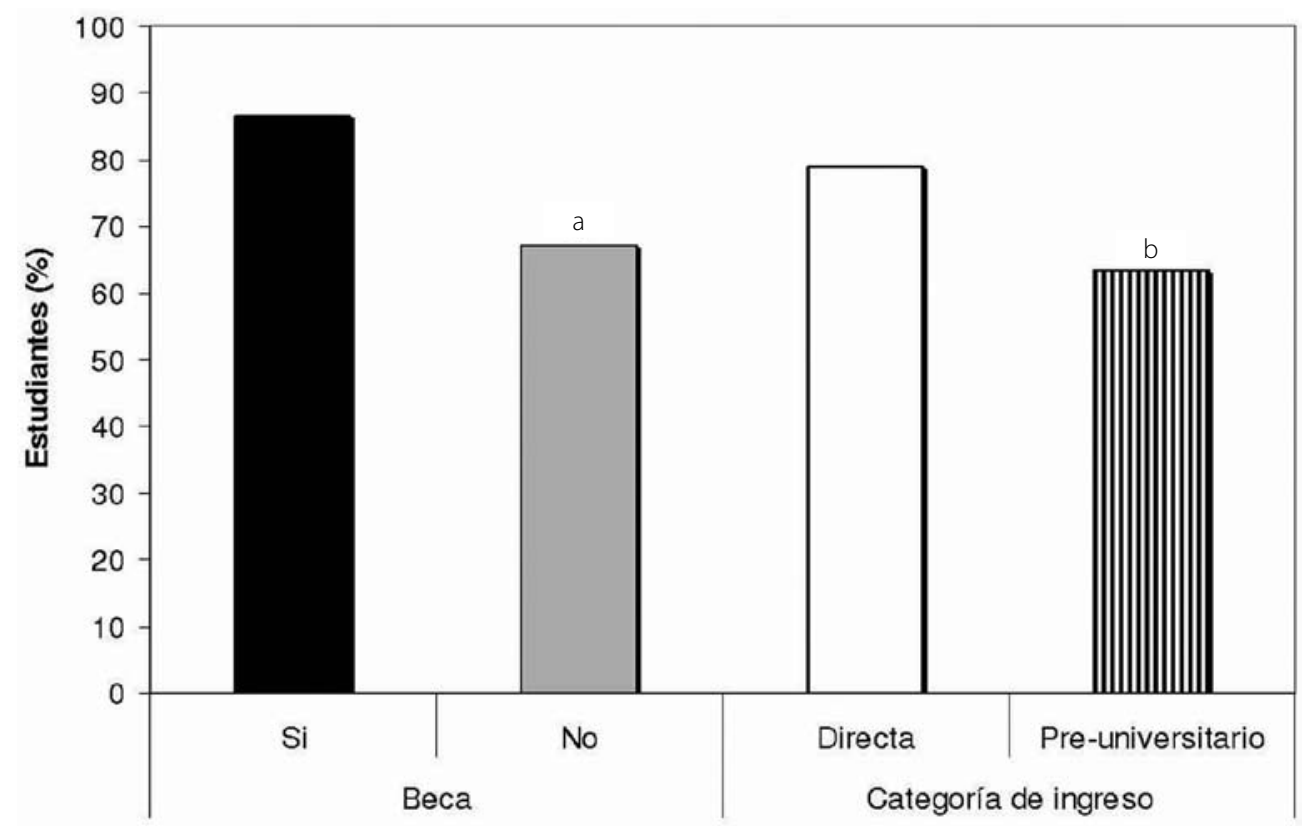

Figura 3. Porcentaje de estudiantes que aprobaron la bioquímica en función de su estatus de becario y de la categoría de ingreso.

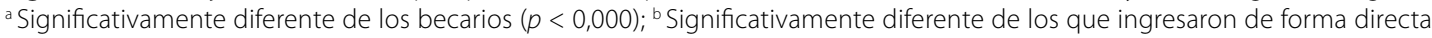
$(p<0,002)$.

va promedio fue muy baja $(3,25 \pm 0,56)$ debido principalmente a los resultados deficientes en las primeras evaluaciones; posiblemente esto pudo ser consecuencia de que los estudiantes se enfrentaron por vez primera a una metodología de enseñanza-aprendizaje que les exigió una participación activa en el proceso de apropiación del conocimiento, en contraste con las metodologías tradicionales de aprendizaje pasivo y las evaluaciones de confrontación memorística, con ausencia de análisis, a las cuales venían acostumbrados por ser las que generalmente se practican en un gran número de establecimientos de educación media en Colombia. Estos resultados indican que, en promedio, tomando como criterio de calidad y buen rendimiento calificaciones de 4,0 puntos o más, el desempeño académico del grupo de estudiantes analizados fue bajo.

$\mathrm{Al}$ igual que Edel [5], creemos que los bajos rendimientos y la mala calidad de las promociones podrían explicarse partiendo de la base de la apreciación realizada por los propios estudiantes de niveles educativos medio y superior, acerca de las deficiencias en los fundamentos de su formación, manifestadas en dificultades aca- démicas que van desde una mala ortografía hasta la incapacidad para llevar a cabo procesos de pensamiento elementales, tales como el análisis, la capacidad de síntesis o la toma de decisiones ante diferentes planteamientos, entre otros. Por otra parte, la transición de la educación media a la universidad influye negativamente en el rendimiento académico y, por ello, es imperioso brindar alternativas de apoyo y orientación no sólo durante la fase académica inicial, sino a lo largo de todo el periodo universitario $[13,15]$.

Por ello, en el último período analizado (2008-I), el grupo de profesores diseñó una estrategia de tutoría o acompañamiento académico extraclase, con la cual se buscó que los estudiantes profundizaran en conocimientos básicos de química necesarios para la comprensión de la bioquímica y que se entrenaran en la resolución de problemas [13]; indudablemente, esas estrategias contribuyeron significativamente a mejores resultados en los promedios de calificaciones en ese semestre, comparado con los otros semestres académicos de las diferentes cohortes (Fig. 2).

Estos resultados también confirmaron una apreciación tradicional y empírica de los pro- 
fesores, referente a la mayor calidad, analizada como rendimiento académico, de los grupos que ingresan en los primeros semestres del año, así como una mejor actitud ante la metodología de enseñanza y la construcción de su propio proceso de aprendizaje. Creemos que este fenómeno se relaciona con la posibilidad de ingreso inmediato a la universidad una vez se ha concluido la educación media. Cuando los estudiantes permanecen fuera del medio académico por uno o más semestres, e incluso años, su destreza para enfrentarse a las pruebas académicas disminuye de forma notable posiblemente debido a que han 'olvidado' sus conocimientos, dificultándose su admisión universitaria y su desempeño en el caso de que lo logren. Ha sido evidente además que, en estos segundos semestres del año, ingresan estudiantes con edades superiores (mayores de 25 años) a los que normalmente vienen a los primeros semestres (15-17 años); ahora bien, el retraso en el ingreso a la universidad obedece a diversas razones, entre las cuales están la cobertura limitada que ofrece el Estado, las deficiencias académicas en conocimientos de ciencias naturales e incluso los problemas económicos familiares.

Se han encontrado correlaciones significativas entre la actitud del estudiante hacia el aprendizaje y el rendimiento académico, lo que demuestra una asociación entre dichos parámetros. Igualmente, se ha descrito que la motivación de los estudiantes contribuye a la predicción del éxito escolar por encima de la inteligencia [16,17]. Lo anterior destaca la importancia de desarrollar una buena actitud y motivación hacia el aprendizaje poniendo en marcha acciones educativas por parte de los profesores para lograrlo. La implementación de metodologías de aprendizaje, así como de evaluaciones más activas y constructivas, y una interpretación de la interacción profesor/estudiante como relación educativa y no sólo instructiva -más comprometida por tanto con la formación 'integral' de los estudiantes-, serían necesarias para avanzar en este terreno $[7,13]$. En este sentido, nuestro grupo ha destacado la importancia de utilizar actividades académicas extraclase como estrategia para que el estudiante reconozca críticamente sus debilidades y busque autónomamente soluciones, y para que el tutor trascienda los procesos de aprendizaje de saberes hacia la formación del individuo, de tal forma que la enseñanza se proyecte hacia un proceso realmente educativo [13].
La implementación desde el año 2005 de la nueva metodología de aprendizaje activo, con las estrategias pedagógicas ya mencionadas, específicamente en la cátedra de Bioquímica impartida en el programa de Medicina de la Universidad del Rosario ha permitido llevar a la práctica la filosofía de aprender a aprender y demostrar que la actitud de los estudiantes hacia el aprendizaje de esta disciplina ha mejorado y, por ende, su rendimiento académico; esto ha ejercido un impacto positivo sobre la disminución de las tasas de 'mortalidad académica', llevándolas a valores por debajo de los históricos registrados, incluso al mejor aprovechamiento y desempeño en las asignaturas básico-clínicas y clínicas, según apreciaciones de los mismos estudiantes.

En cuanto a los otros factores analizados en el estudio, es muy interesante resaltar que de acuerdo con los resultados del examen de Estado, la mayoría de los estudiantes $(99,4 \%)$ que ingresan en Medicina en la Universidad del Rosario se encuentran en la categoría definida por el Ministerio de Educación Nacional como 'media', con puntuaciones superiores a 55 puntos, lo que corresponde a un valor por encima de la media para el país. La captación de estudiantes con estos perfiles obedece a políticas institucionales donde prima el criterio de dar la oportunidad a la gran mayoría de estudiantes que proceden de la educación media en esas condiciones. Es muy notorio también que en las áreas que evalúa el ICFES, biología es la del mayor promedio y es lo que quizá potencia la inclinación de los jóvenes a estudiar medicina. Aunque contrario a la creencia de muchos de ellos, no son menos importantes las otras disciplinas de las ciencias naturales y mucho menos las matemáticas, cuyos bajos promedios en el examen estatal podrían estar influyendo en los bajos rendimientos académicos en bioquímica.

Al comparar el rendimiento académico, visto como el éxito o el fracaso, con los resultados del examen de Estado (Tabla IV), se observa claramente la influencia positiva que tienen los resultados obtenidos en las ciencias naturales y las matemáticas, así como en el promedio general, sobre la posibilidad de tener éxito en bioquímica; a mayor solidez en las ciencias naturales, mejor desempeño en las ciencias biomédicas, lo cual permite sugerir la necesidad de evaluar la calidad de los programas de Ciencias Naturales en la educación media y de fortalecerlos en los ciclos básicos de la educación superior. 
Tabla IV. Influencia de la preparación previa en ciencias naturales sobre el rendimiento académico, visto como éxito o fracaso, en la asignatura de bioquímica. Éxito: nota $\geq 3,0$; fracaso: nota $<3,0$. Media del examen del Estado (ICFES).

\begin{tabular}{|c|c|c|c|c|c|}
\hline & Categoría & Media & $\begin{array}{l}\text { Desviación } \\
\text { típica }\end{array}$ & $\begin{array}{l}\text { Error típico } \\
\text { de la media }\end{array}$ & $\begin{array}{l}\text { Sig. (bilateral) } \\
\text { inferior }\end{array}$ \\
\hline \multirow{2}{*}{ Promedio general } & Éxito & 57,8 & 4,687 & 0,213 & \multirow{2}{*}{0,000} \\
\hline & Fracaso & 55,5 & 3,879 & 0,322 & \\
\hline \multirow{2}{*}{ Biología } & Éxito & 60,7 & 6,172 & 0,281 & \multirow{2}{*}{0,002} \\
\hline & Fracaso & 58,8 & 5,704 & 0,474 & \\
\hline \multirow{2}{*}{ Química } & Éxito & 56,2 & 5,823 & 0,265 & \multirow{2}{*}{0,000} \\
\hline & Fracaso & 53,8 & 5,178 & 0,430 & \\
\hline \multirow{2}{*}{ Física } & Éxito & 56,1 & 7,475 & 0,340 & \multirow{2}{*}{0,009} \\
\hline & Fracaso & 54,3 & 6,947 & 0,577 & \\
\hline \multirow{2}{*}{ Matemáticas } & Éxito & 57,7 & 10,180 & 0,463 & \multirow{2}{*}{0,005} \\
\hline & Fracaso & 55,0 & 9,837 & 0,817 & \\
\hline
\end{tabular}

Otro factor relacionado con los indicadores de calidad establecidos por el gobierno colombiano es la categorización que hace el ICFES de los colegios en un rango que va desde bajo hasta muy superior. Esta categorización se basa en un promedio de los resultados del examen de Estado de todos los estudiantes que en el año lo presentan, y evidencia que quienes ingresan en la Universidad del Rosario pueden provenir de colegios clasificados como superior y muy superior (92,9\%); sin embargo, entre ellos, un alto porcentaje se ubica en el rango medio de calificación en los resultados individuales de las pruebas estatales, lo cual demuestra la heterogeneidad de la población admitida. No obstante, los resultados del análisis numérico como el de categorización de las variables dependientes mostró una ligera tendencia, aunque sin significación estadística, como posible factor influyente en el rendimiento académico en bioquímica.

La Universidad del Rosario ofrece dos opciones para entrar a cursar la carrera de Medicina; una es de forma directa, es decir, con base en las puntuaciones del ICFES y una entrevista, y la otra, a través de un curso de preparación profesional o preuniversitario para los aspirantes que presenten deficiencias académicas por sus bajas puntuaciones en el examen del ICFES, pero que tras la entrevista se considere que con un apoyo académico previo pueden nivelar sus conocimientos básicos en Ciencias Naturales, principalmente en favor de un mejor desempeño en el primer semestre de la carrera; la proporción entre los que entran de forma directa o indirecta es aproximadamente de 6 a 1 . Los resultados muestran una diferencia significativa $(p<0,000)$ entre los rangos promedio (Tabla III), lo cual se comprobó al realizar el análisis categorizado del rendimiento académico (Fig. 3); en él, se observó una mayor probabilidad de tener éxito en bioquímica (73\% de estudiantes) si el estudiante entra de forma directa a la universidad, ratificando que estos estudiantes no sólo cumplen con los criterios de selección, sino que por su calidad 
académica previa son capaces de desenvolverse mejor durante su primer período lectivo. Por el contrario, los que entran de forma indirecta presentan una menor probabilidad de tener éxito (58\% de estudiantes) y, por consiguiente, un mayor riesgo de fracasar con respecto a los primeros; este resultado permite concluir que la realización de este tipo de cursos, impartidos por la misma universidad, probablemente no son suficientes para nivelar las deficiencias con las que ingresan estos estudiantes, siendo el análisis de las causas que inciden en estos resultados un aspecto de suma importancia a tener en cuenta en un estudio posterior. Este resultado corrobora la necesidad de revisar los programas de Ciencias Naturales que se están impartiendo en la educación media y darle un mayor énfasis a su aprendizaje, como ya se sugirió.

Dentro de los factores que pueden estar influyendo de alguna manera en el desempeño académico de los estudiantes becados está la presión de mantenerse dentro de los mejores promedios académicos del semestre $(>4,0)$ para poder continuar recibiendo estos beneficios u otros, como es el caso de quienes reciben préstamos de entidades gubernamentales para la cancelación de los gastos de matrícula. El programa de becas de la universidad tiene una gran cobertura; aproximadamente el $50 \%$ de los estudiantes que ingresan en el primer semestre cuentan con algún tipo de beca y por esta razón este factor se sitúa como uno de los más importantes porque, como se mostró, tiene una marcada influencia en el rendimiento académico. Las diferencias entre el promedio de los rangos en las calificaciones de estos estudiantes (Tabla III), así como el porcentaje de estudiantes que cursó exitosamente la asignatura (Fig. 3), fueron estadísticamente significativos. Una duda importante que surge de estos resultados es si el mejor desempeño de los estudiantes becados se relaciona con su interés por el conocimiento y su propia formación, o si, por el contrario, la mayor motivación está soportada por la necesidad de mantener la beca para subsanar sus necesidades económicas. Adicionalmente, podría pensarse, como sugiere Edel, que los resultados académicos de estos estudiantes estén mas asociados al mayor esfuerzo por mantenerse en dicho promedio que a las habilidades o capacidades en el desarrollo de sus actividades académicas [5].

Aunque el análisis estadístico de la comparación de los grupos que provienen de lugares geográficos diferentes a la ubicación de la universidad con los que viven en igual localidad que ésta no mostró diferencias significativas, el hecho de proceder de una ciudad diferente al sitio de ubicación de la universidad permite pensar que ese porcentaje de estudiantes $(20 \%)$ necesite un período de adaptación más prolongado debido al desarraigo familiar y social al que se ven sometidos, y que por ello se vean en desventaja con aquellos que no están obligados a tal desplazamiento geográfico. Estos últimos resultados coinciden con los de Vélez y Roa [11], quienes argumentan que los factores demográficos no tienen incidencia significativa sobre el rendimiento académico, comprobándose en este caso, específicamente, en bioquímica.

Finalmente puede decirse que, aunque en el rendimiento académico influyen diversos factores, entre los cuales están los analizados en este trabajo, existen otros de gran importancia como los relacionados con el individuo mismo y, desde luego, con los sistemas de aprendizaje que se utilicen para la apropiación de conocimientos, que merecen ser analizados por quienes ejercemos la labor docente, sin temor a reconocer las limitaciones que imponen los sistemas educativos vigentes y la misma tradición, con el fin de alcanzar los cambios exigidos por las nuevas generaciones [14].

Quizá los límites impuestos por la misma tradición, representados ampliamente por las metodologías de aprendizaje pasivo, y la falta de políticas nacionales y desde luego institucionales que apoyen decididamente los cambios pedagógicos en favor de una verdadera apropiación del conocimiento son los responsables de que estudios como éste aparezcan como casos puntuales y aislados y de que una dinámica programada de comparaciones y análisis mas amplios entre grupos étnicos, por ejemplo, entre disciplinas, etc., no pueda plantearse todavía.

\section{Bibliografía}

1. Benítez M, Giménez M, Osicka R. Las asignaturas pendientes y el rendimiento académico: ¿existe alguna relación? URL: http://www.unne.edu.ar.

2. Jiménez M. Competencia social: intervención preventiva en la escuela. Infanc Soc 2000; 24: 21-48.

3. De Spinola H. Rendimiento académico y factores psico- 
sociales en los ingresantes a la carrera de medicina-UNNE. Revista Paraguaya de Sociología 1990; 78: 143-67.

4. Ruiz E, Ruiz G, Odstrcil M. Metodología para realizar el seguimiento académico de alumnos universitarios. RIE 2007. URL: http://www.rieoei.org/deloslectores/ 1590Ruiz.pdf.

5. Edel R. El rendimiento académico: concepto, investigación y desarrollo. REICE 2003; 1: 1-15. URL: http:// www.ice.deusto.es/rinace/reice/volln2/Edel.pdf.

6. Vicente I. Análisis de las calificaciones escolares como criterio de rendimiento académico. URL: http://www3. usal.es/inico/investigación/jornadas/jornada2/comun/ c17.html.2000.

7. Gargallo B, Pérez C, Serra B, Sánchez F, Ros I. Actitudes ante el aprendizaje y rendimiento académico en los estudiantes universitarios. RIE 2007; 42. URL: http://www. rieoei.org/investigacion/1537Gargallo.pdf.

8. Chain R, Cruz-Ramírez N, Martínez-Morales M. Jácome N. Examen de selección y probabilidades de éxito escolar en estudios superiores. Estudio en una universidad pública estatal mexicana. REDIE 2003; 5. URL: http:// redie.uabc.mx/vol5nol/contenido-chain.html.

9. Tirado F, Backhoff E, Larrazolo N, Rosas M. Validez predictiva del examen de habilidades y conocimientos básicos (EXHCOBA). Revista Mexicana de Investigación Educativa 1997; 2: 67-84. URL: http://www.comie.org. $\mathrm{mx} / \mathrm{v} 1 /$ revista/portal.php? $\mathrm{idm}=\mathrm{es} \& \mathrm{sec}=\mathrm{sc} 03 \& \& \mathrm{sub}=\mathrm{sb}$ b\&criterio $=\operatorname{art} 00213$.
10. Goleman D. La inteligencia emocional: por qué es más importante que el cociente intelectual. Barcelona: Ediciones B; 2004.

11. Vélez A, Roa C. Factores asociados al rendimiento académico en estudiantes de medicina. Educ Med 2005; 8: 74-82.

12. Rojas MO, Del Riesgo L, Garzón R. 'Bioquímica I: de la estructura a la función'. La pregunta como base de la investigación científica. Orientaciones para el aprendizaje de la Bioquímica. Colección Lecciones de Medicina. Bogotá: Universidad del Rosario; 2007.

13. Rojas MO, Garzón R, Del Riesgo L, Pinzón M, Salamanca AL, Pabón L. Estrategias pedagógicas como herramienta educativa: 'la tutoría y el proceso formativo de los estudiantes'. RIE 2009; 50. URL: http://www.rieoei. org/deloslectores/3007Rojas.pdf.

14. Rojas M, Garzón R, Del Riesgo L. ¿Continuamos informando o asumimos el cambio? Rev Cien Salud (Especial) 2006; 4: 177-85.

15. Edel R. Factores asociados al rendimiento académico. RIE 2003. URL: http://www.rieoei.org/investigacion 1512Edel.pdf.

16. Steinmayr R, Spinath B. The importance of motivation as a predictor of school achievement. Learning and Individual Differences 2009; 19: 80-90.

17. Spinath B, Spinath F, Harlaar N, Plomin R. Predicting school achievement from general cognitive ability, selfperceived ability, and intrinsic value. Intelligence 2006; 34: 363-74. 